\title{
The influence of defects and composition on the electronic structure and magnetic properties of shape memory Heusler alloys
}

\author{
S. Kulkova ${ }^{\text {a1 }}$, S. Eremeev ${ }^{1}$, Q.M. Hu ${ }^{2}$, C.M. Li $^{2}$ and R. Yang ${ }^{2}$ \\ ${ }^{1}$ Institute of Strength Physics and Materials Science SB RAS, 634021Tomsk, Russia \\ ${ }^{2}$ Shenyang National Laboratory for Materials Science, Institute of Metal Research, Chinese Academy of \\ Sciences, 110016 Shenyang, China
}

\begin{abstract}
The electronic structure and magnetic properties in the $\mathrm{Ni}_{2} \mathrm{MnZ}$ series, where $\mathrm{Z}=\mathrm{Al}, \mathrm{Ga}, \mathrm{In}, \mathrm{Sn}, \mathrm{Sb}$, are studied using the first first-principles methods within density functional theory. The formation energies of several kinds of defects (atomic swaps, antisites. vacancies) are estimated. It is shown that Ni-antisites on the Mn sublattice have the lowest formation energy in the investigated series.
\end{abstract}

\section{Introduction}

The ferromagnetic Heusler alloys are studied intensively for last decade due to their interesting magneto-elastic properties, in particular, shape memory effect, magnetic field induced superelasticity, etc. They are very attractive for applications as different kind of actuators, sensors, magnetic micro-electro-mechanical systems, for recording and storage of information. These alloys undergo martensitic transformation in the tetragonal structures with different c/a ratio. Depending on alloy composition, the martensitic phase occurs as modulated $2 \mathrm{M}, 3 \mathrm{M}, 5 \mathrm{M}, 7 \mathrm{M}$ structures. The phase transitions in ferromagnetic alloys depend strongly on the structural defects or deviation from stoichiometric composition, and are connected with the peculiarities of the electronic structure. The most experimentally and theoretical investigated Heusler alloy is $\mathrm{Ni}_{2} \mathrm{MnGa}$. Numerous experiments have shown that martensitic temperature $\left(T_{M}\right)$ is very sensitive to the composition of the alloy [1-5]. For example, $\mathrm{T}_{\mathrm{M}}$ in the case of $\mathrm{Ni}_{2.18} \mathrm{Mn}_{0.82} \mathrm{Ga}$ is $340 \mathrm{~K}$ whereas the $\mathrm{T}_{\mathrm{M}}$ for $\mathrm{Ni}_{2.16} \mathrm{MnGa}_{0.84}$ reaches $532 \mathrm{~K}$. It was shown that the martensitic temperature and Curie temperature $\left(\mathrm{T}_{C}\right)$ linearly increases/decreases with Ni content in $\mathrm{Ni}_{2+\mathrm{x}} \mathrm{Mn}_{1-\mathrm{x}} \mathrm{Ga}$ alloys [3]. The off-stoichiometry manifests itself by the existence of point defects in the lattice. Therefore, investigations on the point defects in Heusler alloys are critical to the understanding of the composition dependence of their properties. On the other hand, since Heusler alloys have the similar lattice parameters to those of many semiconductors of III-V group, they can epitaxially grow on the top of them. Substrate temperature can influence the interfacial reactions, crystal quality, magnetic properties, atomic ordering of alloys. In order to understand the influence of different defects on the electronic and magnetic properties of Heusler alloys, their thin films and interfaces with semiconductors, we need to study first of all the formation energies of defects such as antisites, swaps (interchange of atoms), and vacancies.

In our previous paper [6] we estimated the formation energies of several kinds of defects in the case of $\mathrm{Ni}_{2} \mathrm{MnGa}$ and $\mathrm{Ni}_{2} \mathrm{FeGa}$. For both alloys the smallest formation energies were obtained for Ni antisite defect on the Mn sublattice. In the present paper, we extend our analysis by systematically investigating the formation of defects (antisites, atomic swaps, vacancies) in the series of alloys $\mathrm{Ni}_{2} \mathrm{MnZ}$, where $\mathrm{Z}$ is $\mathrm{Al}, \mathrm{Ga}, \mathrm{In}, \mathrm{Sn}, \mathrm{Sb}$.

\section{Method of calculation}

Atomic and electronic structures of both ideal and defective Heusler alloys were studied using density functional theory, the projected-augmented-wave method [7] implemented in the VASP code [8-11] within generalized gradient approximation (GGA) [12] for the exchange-correlation functional. The calculations of electronic structure were performed for $2 \times 2 \times 2$ times L2 ${ }_{1}$ unit lattice. The lattice constant was fixed to the calculated bulk equilibrium value but the positions of atoms near defects were optimized using Newton dynamics. A $4 \times 4 \times 4$

\footnotetext{
a e-mail: kulkova@ms.tsc.ru
}

This is an Open Access article distributed under the terms of the Creative Commons Attribution-Noncommercial License (http://creativecommons.org/licenses/by-nc/3.0/), which permits unrestricted use, distribution, and reproduction in any noncommercial medium, provided the original work is properly cited. 
mesh of $k$ points was used for Brillouin zone integration. A plane waves cutoff was set to $300 \mathrm{eV}$. The total energies were converged up to $10^{-4} \mathrm{eV}$. The defect formation energy was estimated using equation

$$
\Delta E=E_{\text {def }}-E_{i d}+n_{X} \mu_{X}+n_{Y} \mu_{Y}+n_{Z} \mu_{Z}
$$

where $E_{d e f}$ and $E_{i d}$ is the total energies of the defective and ideal unit cell, respectively; $n_{\mathrm{i}}$ is the number of atoms transferred to or from a chemical reservoir that has a characteristic energy $\mu_{\mathrm{i}}$.

\section{Results and discussion}

\subsection{Point defect formation energy}

The calculated formation energies of several kinds of defects in the five different matrixes are given in Table 1. As it is seen from Table 1 the $\mathrm{Ni}$ antisite defect on the $\mathrm{Mn}$ sublattice has the smallest value in the $\mathrm{Ni}_{2} \mathrm{MnZ}_{\text {series }}$ $(\Delta \mathrm{E}=0.26-0.37 \mathrm{eV})$. This suggests that this defect is more preferable to be formed during alloy growth. The formation energy of $\mathrm{Mn}$ antisites on the $\mathrm{X}$ sublattice, which were found to be more preferable for $\mathrm{Co}_{2} \mathrm{MnZ}$ alloys in $[6,13]$, is of $\sim 0.1-0.3 \mathrm{eV}$ larger than that for $\mathrm{Ni}$ antisites. Generally speaking, for $\mathrm{Z}=\mathrm{Al}, \mathrm{Ga}$, and In, the vacancy formation energies are larger than those of the antisites (Table 1), indicating that the excess atoms in theses alloys exist as antisite defects. Howevere, for $\mathrm{Z}=\mathrm{Sn}$ and $\mathrm{Sb}$, some of the vacancy formation energies (especially for the Ni-vacancies) are smaller than those of the antisite formation energies. Therefore, for $\mathrm{Z}=\mathrm{Sn}$ and $\mathrm{Sb}$, the off-stoichiometry (e.g., the $\mathrm{Ni}$ deficiency) can be realized by the formation of vacancies. In additional we estimated also $\mathrm{Mn}$ and $\mathrm{Ni}$ antisites on $\mathrm{Z}$ sites. One can see from Table 1 that $\mathrm{Z}$ antisites have the smallest formation energies for the first three alloys in the series whereas the energies of $\mathrm{Mn}$ antisites on $Z$ sites are lowest for last alloys in this series. The formation of $\mathrm{Ni}$ antisites on $\mathrm{Z}$ sites is less favorable among considered antisites defects. The formation energy of Mn-Z swaps is smaller at the beginning of the series but it is substantial higher for $\mathrm{Ni}_{2} \mathrm{MnSn}$ and $\mathrm{Ni}_{2} \mathrm{MnSb}$. The formation energies of $\mathrm{Mn}-\mathrm{Ni}$ swaps do not differ significantly in the series as in the previous case, moreover it decreases with increase of the number of the Zelement in the periodic table. It is possible to suggest that the sizeable effect can be responsible for the decrease of $\mathrm{Mn}-\mathrm{Ni}$ formation energy. In general, the estimation of the formation energies of considered defects shows that the situation is not so simple in $\mathrm{Ni}_{2} \mathrm{MnZ}$ series and several kinds of defects can be formed simultaneously.

Table 1. Formation energies (in $\mathrm{eV}$ ) of considered defects.

\begin{tabular}{|c|c|c|c|c|c|c|c|c|c|c|}
\hline & $\begin{array}{c}\text { Mn-Z } \\
\text { swap }\end{array}$ & $\begin{array}{c}\text { Ni-Mn } \\
\text { swap }\end{array}$ & $\begin{array}{c}\text { Mn antisite } \\
\text { on Ni }\end{array}$ & $\begin{array}{c}\text { Ni antisite } \\
\text { on Mn }\end{array}$ & $\begin{array}{c}\text { Mn antisite } \\
\text { on Z }\end{array}$ & $\begin{array}{c}\text { Ni antisite } \\
\text { on Z }\end{array}$ & $\begin{array}{c}\text { Z antisite } \\
\text { on Mn }\end{array}$ & $\begin{array}{c}\text { Mn- } \\
\text { vac }\end{array}$ & $\begin{array}{c}\text { Ni- } \\
\text { vac }\end{array}$ & $\begin{array}{c}\text { Z- } \\
\text { vac }\end{array}$ \\
\hline $\mathrm{Ni}_{2} \mathrm{MnAl}$ & 0.52 & 0.91 & 0.69 & 0.28 & 1.61 & 1.72 & -0.87 & 1.68 & 1.48 & 3.18 \\
\hline $\mathrm{Ni}_{2} \mathrm{MnGa}$ & 0.59 & 0.70 & 0.48 & 0.27 & 1.63 & 1.74 & -0.79 & 1.36 & 1.12 & 3.28 \\
\hline $\mathrm{Ni}_{2} \mathrm{MnIn}$ & 0.56 & 0.92 & 0.64 & 0.37 & 0.39 & 0.84 & 0.29 & 1.58 & 0.70 & 2.29 \\
\hline $\mathrm{Ni}_{2} \mathrm{MnSn}$ & 1.30 & 0.80 & 0.66 & 0.29 & 0.69 & 1.16 & 1.12 & 1.04 & 0.40 & 2.59 \\
\hline $\mathrm{Ni}_{2} \mathrm{MnSb}$ & 1.53 & 0.57 & 0.36 & 0.26 & 0.36 & 0.87 & 2.02 & 0.39 & 0.11 & 2.43 \\
\hline
\end{tabular}

The dependence of formation energy on volume is given in Fig. 1. It is seen that $\Delta \mathrm{E}$ of $\mathrm{Ni}$ antisites on $\mathrm{Mn}$ sites increases approximately linearly with the volume increase. The opposite trend is found for $\mathrm{Mn}-\mathrm{Z}$ swaps. In the case of $\mathrm{Ni}-\mathrm{Mn}$ swaps $\Delta \mathrm{E}$ increases with increase of volume for $\mathrm{Ni}_{2} \mathrm{MnGa}$ and $\mathrm{Ni}_{2} \mathrm{MnSn}_{\mathrm{Sut}}$ it is almost unchanged for $\mathrm{Ni}_{2} \mathrm{MnIn}$ and it reaches maximal value at normal condition in the case of $\mathrm{Ni}_{2} \mathrm{MnAl}$. We studied also the influence of tetragonal distortion on the Ni-antisites formation energy for $\mathrm{Ni}_{2} \mathrm{MnGa}_{\text {. It was }}$ found that $\Delta \mathrm{E}$ is decreased only slightly by $0.05 \mathrm{eV}$, when $0.9<c / a<1.1$.
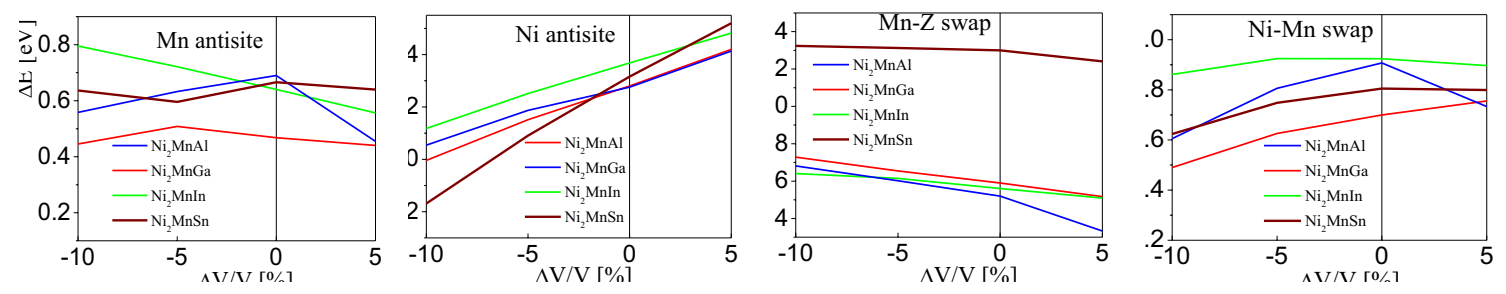

Fig. 1. The volume dependence of defects formation energy for $\mathrm{Ni}_{2} \mathrm{MnZ}$ alloys.

The arrangement of the atoms in the lattice of off-stoichiometric Heusler alloys is of particular interest since both $\mathrm{T}_{\mathrm{M}}$ and $\mathrm{T}_{\mathrm{C}}$ depend on the degree of the $\mathrm{L} 2_{1}$ long range atomic order (site-occupancy) of the alloy which can be changed by the quenching temperature and the subsequent heat treatment [14]. For a offstoichiometric alloy at its thermal equilibrium state, the site-occupancy seems to be straightforward, i.e., the 
excess atoms of the rich component would occupy the sublattice(s) of the deficient one(s). So, in $\mathrm{Ni}_{2-\mathrm{x}} \mathrm{MnGa}_{1+\mathrm{x}}$ alloy, one would expect that, if not considering the existence of vacancies, the excess $\mathrm{Ga}$ atoms take the $\mathrm{Ni}$ sublattice to form Ga antisite defects (so-called "normal" site-occupation). However, we can not rule out the possibility that the excess $\mathrm{Ga}$ atoms take the Mn sublattice (Ga antisite on Mn sublattice); some of the Mn atoms move to the Ni sublattice, i.e., introducing further Mn antisite on Ni sublattice ("abnormal" site-occupation), since $\mathrm{Ga}$ antisite on $\mathrm{Mn}$ sublattice is extremely preferable (with negative formation energy) whereas the formation energy of $\mathrm{Mn}$ antisite on $\mathrm{Ni}$ sublattice is also small. The energetics of the point defects in offstoichiometric $\mathrm{Ni}_{2} \mathrm{MnGa}$ alloys is in agreement with our previous work reported in Ref. [15], where we investigated the site-occupancy of the off-stoichiometric $\mathrm{Ni}_{2} \mathrm{MnGa}$ alloys by the first-principles exact muffin-tin orbital method in combination with coherent potential approximation. In Ref. [15], the "abnormal" siteoccupancy in $\mathrm{Ni}_{2-\mathrm{x}} \mathrm{MnGa}_{1+\mathrm{x}}$ was found to be more stable than the "normal" one.

\subsection{Electronic Density of States}

The electronic structure for both defective and ideal alloys with X, Y, Z antisites defects are given in Fig. 2. Only minor defect-induced changes of total densities of states (DOS) are occurred for all considered alloys in the case of antisites defects. The modification of the DOS structure near the Fermi level $\left(\mathrm{E}_{\mathrm{F}}\right)$ is entirely due to the $\mathrm{Mn}$ antisite d-states. It is interesting to note that for beginning of the Heusler alloys series there is a sharp peak for the majority-spin-states at $\mathrm{E}_{\mathrm{F}}$, as in the case of $\mathrm{Ni}_{2} \mathrm{MnGa}$ in Fig. 2, but it is disappeared for the last two alloys in the series. The second feature at $\sim 1.5 \mathrm{eV}$ for the majority-spin-states is observed for whole series. More pronounced changes in DOS structures for spin-down states below $\mathrm{E}_{\mathrm{F}}$ are connected with Ni antisites on Mnsites.
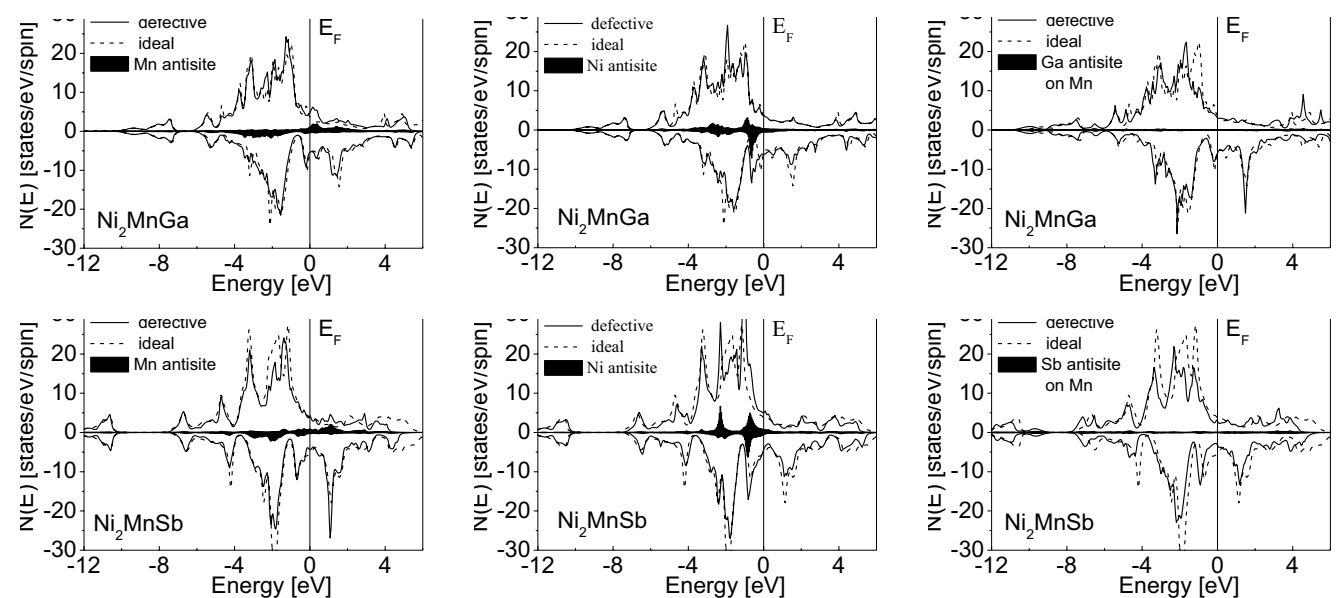

a)

b)

c)

Fig. 2. Total densities of states for ideal and defective $\mathrm{Ni}_{2} \mathrm{MnZ}$ alloys with $\mathrm{Mn}$ antisites on Ni-site (left), $\mathrm{Ni}$ antisites on $\mathrm{Mn}$ site (middle) and $\mathrm{Z}$ antisites on $\mathrm{Mn}$ site (right).

As it is seen from Fig. 2 the Ni antisites induce substantial changes in DOS structure in the case of $\mathrm{Ni}_{2} \mathrm{MnGa}$ (the same is also found for the first alloys in the series) but spin-down states change insignificantly in the case of $\mathrm{Ni}_{2} \mathrm{MnSb}$. The shift of spin-down states below the Fermi level is connected with filling of the Niband due to an increase of Ni content. For the last two alloys of this series the shift of spin-down states is mostly occurred due to increase of the valence electrons in the system when $\mathrm{Z}$ is $\mathrm{Sn}$ or $\mathrm{Sb}$. The calculation of the local DOS showed that $\mathrm{Z}$ antisites on Mn sites lead to decrease of both spin-up and spin-down Mn states.
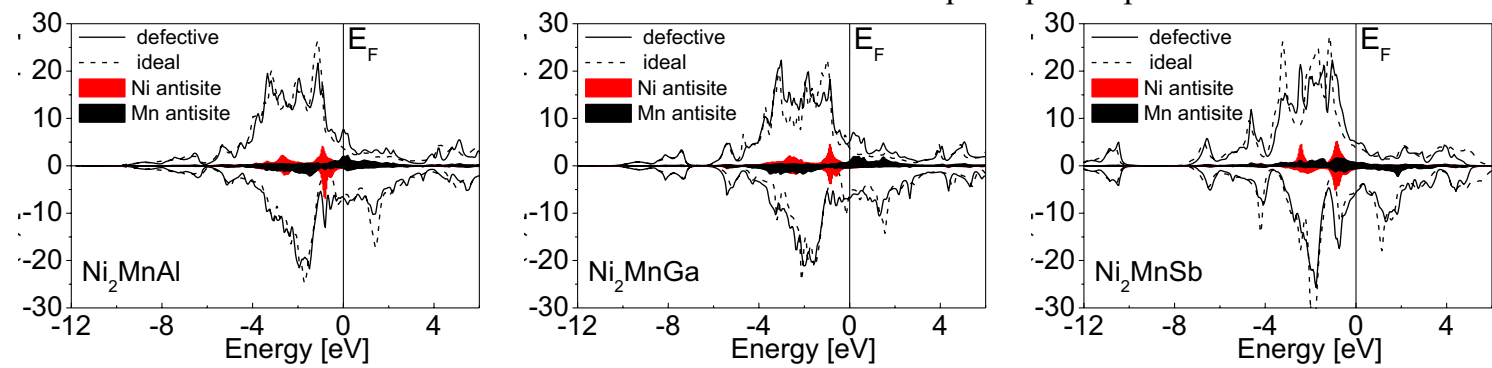

Fig. 3. Total DOS for ideal and defective $\mathrm{Ni}_{2} \mathrm{MnZ}$ alloys with $\mathrm{Ni}-\mathrm{Mn}$ atomic swaps. 

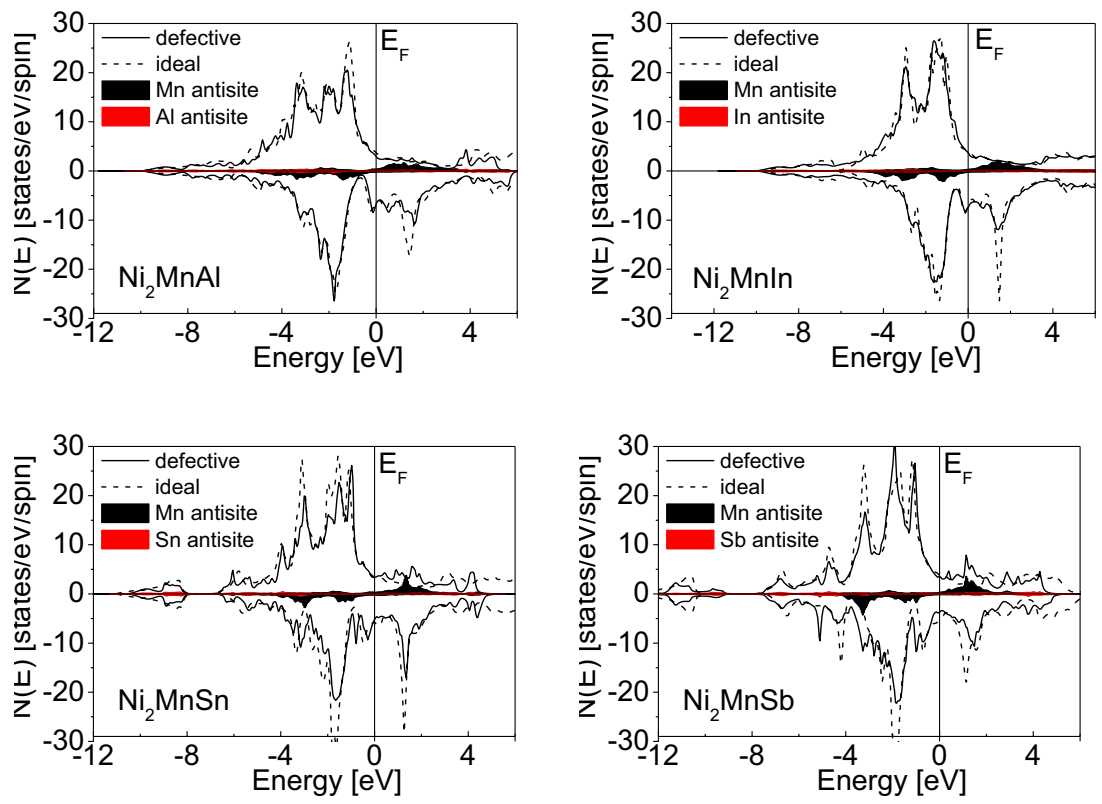

Fig. 4. Total DOS for ideal and defective $\mathrm{Ni}_{2} \mathrm{MnZ}$ alloys with $\mathrm{Mn}-\mathrm{Z}$ atomic swaps.

Actually the same changes below $\mathrm{E}_{\mathrm{F}}$ in the DOS structure as in the case of both $\mathrm{Ni}(\mathrm{Z})$ and Mn antisites one can see in the case of Mn-Ni (Fig. 3) or Mn-Z swaps (Fig. 4). The existence of vacancies on the different sublattice leads to shift of $\mathrm{E}_{\mathrm{F}}$ to lower energy due to decrease of valence electrons. For example, the Ni deficiency influences the DOS near $\mathrm{E}_{\mathrm{F}}$ and leads to the narrowing of $\mathrm{Ni}$ subbands. This results in the delocalization of Mn states. The Mn deficiency leads to shift of Mn spin-down states towards to the Fermi level that influences also DOS at $\mathrm{E}_{\mathrm{F}}$. It should be noted that we can also influence the spin-down states near $\mathrm{E}_{\mathrm{F}}$ by alloying on Mn sublattice. As it was shown in [16] Yan-Teller mechanism can be responsible for the phase transitions in $\mathrm{Ni}_{2} \mathrm{MnGa}$. The splitting of DOS near the Fermi level at the tetragonal distortions is mainly due to Ni spin-down states. So, the change of Ni concentration due to presence of defects or alloying allows influencing on the phase transformations in these alloys.

\subsection{Magnetic moments}

Schematic view of the region close to Mn-antisite and Mn-Z atomic swap is given in Fig. 5 in comparison with the same region in the ideal $\mathrm{Ni}_{2} \mathrm{MnGa}$ alloy. The magnetic properties of the Heusler alloys involved in the paper are mainly ascribed to the local moments of Mn atoms. Therefore, it is important to know how the magnetic moments of the Mn atoms behave in off-stoichiometric alloys.

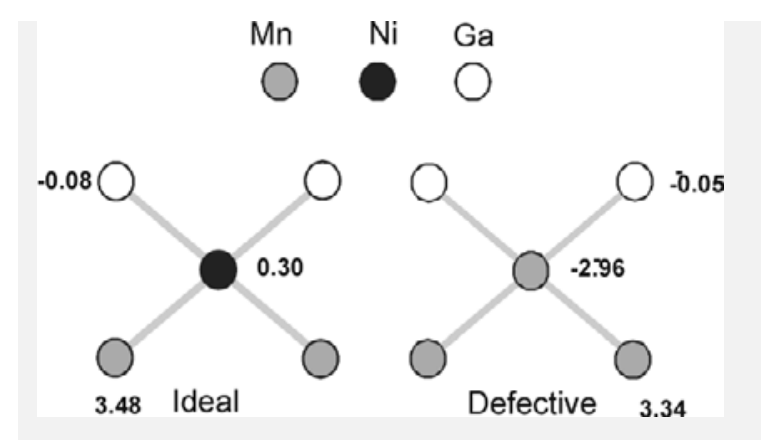

a)

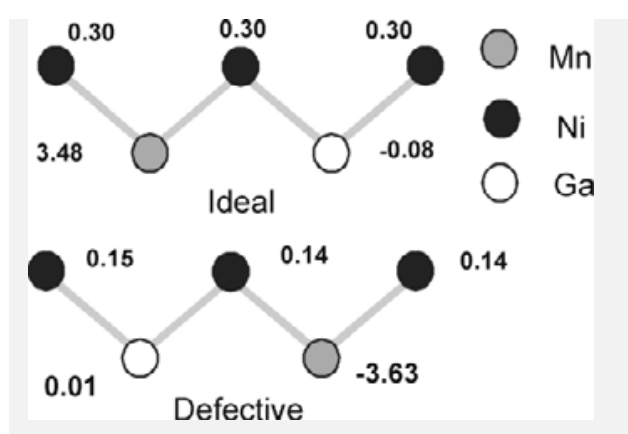

b)

Fig. 5. Atomic structure of the Mn antisite on Ni-site (a), Mn-Ga swap (b) and magnetic moments at atoms near defects for ideal and defective $\mathrm{Ni}_{2} \mathrm{MnGa}$.

We note that a Mn atom changes its magnetic moment when it is located on different sublattices. For $\mathrm{Ni}_{2} \mathrm{MnGa}$ it is $3.48 \mu_{\mathrm{B}},-2.96 \mu_{\mathrm{B}}$, and $-3.7 \mu_{\mathrm{B}}$, on $\mathrm{Mn}$, Ni and $\mathrm{Ga}$ sublattices, respectively. So, the nearest neighboring $\mathrm{Mn}$ atoms, when they occupy $\mathrm{Ni}$ or Ga sublattices prefer to have magnetic moments with opposite orientations. Ni and $\mathrm{Ga}$ atoms on $\mathrm{Mn}$ site are also spin polarized with local magnetic moments of $0.26 \mu_{\mathrm{B}}$ and $0.02 \mu_{\mathrm{B}}$, which are approximately the same when they locate at own sites. With increase of $\mathrm{Z}$ number the 
magnetic moments of Ni slightly decreases. The magnetic moment at $\mathrm{Sn}$ and $\mathrm{Sb}$ has the same orientation as it at Mn site but actually it is negligible $0.003 \mu_{\mathrm{B}}$ and $0.024 \mu_{\mathrm{B}}$, respectively. It should be noted that magnetic moments of a $\mathrm{Ni}$ atom on the $\mathrm{Z}$ sublatice and a $\mathrm{Z}$ atom on the $\mathrm{Ni}$ sublattice are also negligible. We studied also magnetic properties of $\mathrm{Ni}_{2} \mathrm{Mn}_{1 \pm x} \mathrm{Z}_{1 \mp \mathrm{x}}$ alloys. It was found that tetragonal distortion $\sim 1-2 \%$, is observed with increase of $\mathrm{Z}$ concentration, and antiferomagnetic ordering is more preferable in this case as for NiMn. The increase of the Mn concentration leads also to increase of total magnetic moment $\left(\mu=1.6 \mu_{\mathrm{B}} /\right.$ atom at 12.5 at..\% $\mathrm{Al}$ or $\mathrm{Ga}$ ). In general, the Mn spin magnetic moment increases with increase of a number of valence electrons of $\mathrm{Z}$ element. In spite of $\mathrm{Mn}$ mainly contributes to the total magnetic moment, namely Ni determines the behavior of total magnetic moment in $\mathrm{Ni}_{2} \mathrm{Mn}_{1 \pm \mathrm{x}} \mathrm{Z}_{1 \mp \mathrm{x}}$ alloys upon distortions because the tetragonal distortions influence nonmonotonically the magnetic moment at $\mathrm{Ni}$ atoms. Actually the alloying by Fe on the Mn sublattice in the case of $\mathrm{Ni}_{2} \mathrm{Mn}_{1-\mathrm{x}} \mathrm{Fe}_{\mathrm{x}} \mathrm{Z}$ leads to decrease of total spin moment of alloys but we did not found sharp increase of magnetic moment at $\mathrm{Fe}$ up to $\sim 5 \mu_{\mathrm{B}}$ at low concentration of $\mathrm{Fe}$ in $\mathrm{Ni}_{2} \mathrm{Mn}_{1-\mathrm{x}} \mathrm{Fe}_{\mathrm{x}} \mathrm{Sn}$ as in [17]. The Fe moment is equal to $\sim 2.89 \mu_{\mathrm{B}}$ in $\mathrm{Ni}_{2} \mathrm{Mn}_{1-\mathrm{x}} \mathrm{Fe}_{\mathrm{x}} \mathrm{Sn}$ and $\mathrm{Ni}_{2} \mathrm{Mn}_{1-\mathrm{x}} \mathrm{Fe}_{\mathrm{x}} \mathrm{Ga}$ but it is larger than that in pure $\alpha-\mathrm{Fe}$. Almost the same result was obtained in the alloys with In or Sb. The magnetic moment at Ni atoms depends insignificantly on the concentration of $\mathrm{Fe}$ on the Mn sublattice but it depends more substantial on $\mathrm{Z}$ element.

\section{Conclusion}

$\mathrm{Ab}$-initio calculations of the electronic structure, magnetic properties were performed for both ideal and defective $\mathrm{Ni}_{2} \mathrm{MnZ}$ alloys. The estimation of defects formation energies showed that Ni-antisites on $\mathrm{Mn}$ sites are more preferable defect in $\mathrm{Ni}_{2} \mathrm{MnZ}$ series than $\mathrm{Mn}$ antisites on $\mathrm{Ni}$ sites. In the case of $\mathrm{Al}, \mathrm{Ga}, \mathrm{In} \mathrm{Z}$-antisites on $\mathrm{Mn}$ sites can be also easily formed. In general, $\mathrm{Mn}-\mathrm{X}$ and $\mathrm{Mn}-\mathrm{Z}$ swaps defects are found to be less preferable in this series. Both antisites and swaps defects influence strongly magnetic properties of atoms around defects. Since the vacancy formation energies are larger than those of the antisites or swaps this allows to conclude that the excess atoms in $\mathrm{Ni}_{2} \mathrm{MnZ}$ series can exist as antisite defects. In general, obtained results allow us to understand the microscopic origin of the change of electronic and magnetic properties due to structural defects in alloys.

The authors acknowledge the financial support from the NSFC under Grants No. 50631030, No. 50871114, and No. 08-0292201a RFBR-NSFC project for the China-Russian cooperation.

\section{References}

[1] P. J. Webster, K. R. A. Ziebeck, S. L. Town, M. S. Peak, Philos. Mag. B 49, 295 (1984)

[2] A. Chernenko, J. Pons, C. Segui, E. Cesari, Acta Mater. 50, 53 (2002)

[3] V.V. Khovailo, V. Novosad, T.Takagi, D.A. Filippov, R.Z. Levitin, A.N. Vasil'ev, Phys. Rev. B 70, 174413 (2004)

[4] M. Ohtsuka, M. Matsumoto, K. Itagaki, Mater. Sci. Eng., A 438-440, 935 (2006)

[5] Y. Li, Y. Xin, C. Jiang, H. Xu, Mater. Sci. Eng., A 438-440, 978 (2006)

[6] S.E. Kulkova, S.V. Eremeev, S. S. Kulkov, V.A. Skripnyak. Mat. Science and Eng. A. 481-482, 209 (2008)

[7] P.E. Blöchl, Phys. Rev. B 50, 17953 (1994).

[8] G. Kresse, J. Hafner, Phys. Rev. B 47, 558 (1993)

[9] G. Kresse, J. Hafner, Phys. Rev. B 49, 14251 (1994)

[10] G. Kresse, J. Furthmüller, Comput. Mat. Sci. 6, 15 (1996)

[11] G. Kresse, J. Furthmüller, Phys. Rev. B 54, 11169 (1996)

[12] J. P. Perdew, K. Burke, M. Ernzerhof, Phys. Rev. Lett. 77, 3865 (1996)

[13] S. Picozzi, A. Continenza, Phys. Rev. Lett. 69, 094423 (2004)

[14] V. Sanches-Alarcos, V. Recarte, J.I. Perez-Landazabal, G.I. Cuello, Acta Mater. 55, 3883 (2007)

[15] Q.M. Hu, C.M. Li, R. Yang, S.E. Kulkova, D. I. Bazhanov, B. Johansson, L. Vitos, Phys. Rev. B 79, $144112(2009)$

[16] A. Ayuela, J. Enkovaara, R.M. Nieminen. J. Phys.: Condens. Matter 14, 5325 (2002)

[17] C.C.M. Cambell, J.A. Cameron. J. Phys. F: Met. Phys. 8, 1591 (1978) 